\title{
Effect of Aluminum Incorporation into Mesoporous Aluminosilicate Framework on Drug Release Kinetics
}

\author{
Raul-Augustin Mitran, ${ }^{1}$ Daniela Berger, ${ }^{2}$ Jeanina Pandele-Cusu, $^{1}$ and Cristian Matei ${ }^{2}$ \\ 1 "Ilie Murgulescu" Institute of Physical Chemistry, Romanian Academy, 202 Splaiul Independentei, 060021 Bucharest, Romania \\ ${ }^{2}$ Faculty of Applied Chemistry and Material Science, University "Politehnica" of Bucharest, 1-7 Polizu Street, 011061 Bucharest, Romania \\ Correspondence should be addressed to Cristian Matei; cristi_matei@yahoo.com
}

Received 6 March 2017; Revised 17 May 2017; Accepted 8 June 2017; Published 27 July 2017

Academic Editor: Miguel A. Correa-Duarte

Copyright (c) 2017 Raul-Augustin Mitran et al. This is an open access article distributed under the Creative Commons Attribution License, which permits unrestricted use, distribution, and reproduction in any medium, provided the original work is properly cited.

\begin{abstract}
Mesoporous silica materials are promising nanocarriers for the development of drug delivery systems. In this study, the influence of pore size, volume, surface area, and doping the silica framework on the release kinetics of a model drug, metoprolol, has been studied. $20 \%$ or $50 \%$ wt. therapeutic agent was loaded into the carrier mesopores through incipient wetness impregnation. The carriers and drug-loaded samples have been characterized by small- and wide-angle X-ray diffraction, FT-IR spectroscopy, scanning electron microscopy, and nitrogen adsorption-desorption isotherms. The in vitro release profiles have been fitted using a threeparameter kinetic model and they have been explained in terms of the release rate during the burst and sustained release stages and the fraction of drug molecules released during the burst stage. The silica framework doping with aluminum was found to decrease the amount of drug released in the burst stage, without affecting the other kinetic parameters. The therapeutic agent release rates depend mainly on the pore size and volume of the mesoporous carriers and drug-loaded samples.
\end{abstract}

\section{Introduction}

The development of novel drug delivery systems (DDS), which can be adjusted to individual needs, is receiving a great deal of attention lately. Such systems must be biocompatible and able to load a significant amount of therapeutic agent and to release it following a predetermined time profile. Mesoporous silica nanomaterials (MSN) are currently studied for drug delivery applications due to their biosafety $[1,2]$, high adsorption capacity [3-5], and possibility of tailoring the release profiles based on the interactions between the guest drug molecules and silica pore surface [6-8]. The most common methods of modifying the MSN properties include functionalization with organic groups [9-13], doping the silica framework with different atoms [14-17], or changing the morphologic and textural MSN properties, such as particle shape, pore size, specific surface area, total pore volume, or the pore arrangement and geometry [18-25]. Drug release from mesoporous carriers is a complex process, influenced by the structure of the therapeutic agent, the release medium, and the properties of the silica matrix [26-28]. Nonetheless, it is possible to quantify the influence of the carrier properties on the drug release process by using appropriate kinetic models to describe the release process. Such a promising model assumes that the drug release process consists of equilibrium between drug adsorption onto the nanocarrier surface and desorption into the mesopores, followed by transport of the desorbed molecules into the release medium $[29,30]$.

We have investigated the influence of pore size, geometry, and introduction of organic functional groups on the release process of a model drug, metoprolol, in our previous work [31]. It was found that both the textural properties of the carriers and the acidity of the functional group influence the drug release process. In order to more easily distinguish the competing influence of these two types of parameters on the drug release process, it is necessary to be able to change one parameter independent of the others. Silica framework doping with $\mathrm{Al}$ was used in this study to increase the surface acidity without significantly changing the pore size and volume of the mesoporous carriers. Some other porous materials containing aluminum into the silica framework, kaolinite and bentonite, are generally recognized as safe for drug delivery 
applications [32]. MCM-41 and SBA-15 as well as AlMCM-41 and AlSBA-15 have been used as mesoporous carriers.

Metoprolol (MTP), the model drug, has a smaller size than the carrier mesopore diameters (estimated at 1.6 by 0.5 by $0.6 \mathrm{~nm}$ ) and it possesses various functional groups which can participate in carrier-drug supramolecular interactions (hydroxyl, secondary amine, methoxy, and phenoxy). The therapeutic agent is widely used in the treatment of several cardiovascular diseases, acting as a $\beta 1$-receptor blocker [3235]. Herein, the effects of $\mathrm{Al}$ doping and MSN textural parameters on the MTP release kinetics are studied.

\section{Experimental Details}

2.1. Materials. Tetraethylorthosilicate (TEOS, Fluka), AlMCM-41 (Aldrich), 37\% hydrochloric acid solution ( $\mathrm{HCl}$, Sigma-Aldrich), poly (ethylene glycol)-block-poly (propylene glycol)-block-poly(ethylene glycol) (Pluronic ${ }^{\circledR}$ P123, average molecular weight 5800, Sigma-Aldrich), $\mathrm{Al}_{2}\left(\mathrm{SO}_{4}\right)_{3} \cdot 18 \mathrm{H}_{2} \mathrm{O}$ (Merck), myristyltrimethylammonium bromide $\left(\mathrm{C}_{14} \mathrm{TAB}\right.$, Fluka), 25\% $\mathrm{NH}_{3}$ aqueous solution (Scharlau), solvents (Sigma-Aldrich), and metoprolol succinate (MTP, Polipharma Industries) were used as received without further purification. Ultrapure water (Millipore Direct-Q3 UV water purification system with Biopak UF cartridge) was employed in all syntheses and in vitro release experiments.

2.2. Synthesis of Mesoporous Silica Matrices. The detailed synthesis and characterization of MCM-41 and SBA-15 supports and metoprolol-loaded materials were reported in our previous publications $[31,36]$. In brief, MCM-41 was obtained by adding TEOS to an aqueous solution containing $25 \%$ ammonia and $\mathrm{C}_{14} \mathrm{TAB}$. The sol was aged at room temperature for 22 hours, followed by hydrothermal treatment at $150^{\circ} \mathrm{C}$ and $5 \mathrm{~atm}$ for 24 hours. The solids were filtered off, washed, and calcined at $600^{\circ} \mathrm{C}$ for 5 hours. A molar ratio TEOS : $\mathrm{C}_{14}$ TAB : $\mathrm{NH}_{3}: \mathrm{H}_{2} \mathrm{O}$ of $1.0: 0.137: 3.3: 158$ was used. MCM-41 was obtained with $\mathrm{C}_{14} \mathrm{TAB}$ due to the fact that it has closer textural parameters, especially pore size, to commercially available AlMCM-41 (see Table S1 in Supplementary Material available online at https://doi.org/10.1155/2017/ 9864396).

The Al-containing SBA-15 support, denoted as AlSBA15 , was synthesized in acid-free conditions [37], by a slight modification of the procedure reported in [38]. TEOS was added at $40^{\circ} \mathrm{C}$ under stirring to an aqueous solution of $\mathrm{P} 123$ and $\mathrm{Al}_{2}\left(\mathrm{SO}_{4}\right)_{3}$. A TEOS : $\mathrm{P} 123: \mathrm{Al}: \mathrm{H}_{2} \mathrm{O}$ molar ratio of $1.0: 0.0163: 0.1: 184$ was used in this case. The sol was aged under stirring for $24 \mathrm{~h}$ at $40^{\circ} \mathrm{C}$ and then hydrothermally treated at $100^{\circ} \mathrm{C}$ for $24 \mathrm{~h}$ under autogenerated pressure. The solids were recovered by filtration, washed, and calcined at $550^{\circ} \mathrm{C}$ for $6 \mathrm{~h}$ in air, using a heating rate of $1^{\circ} \mathrm{C} / \mathrm{min}$. SBA-15 was similarly obtained, using concentrated $\mathrm{HCl}$ instead of $\mathrm{Al}_{2}\left(\mathrm{SO}_{4}\right)_{3}$. A molar ratio TEOS : $\mathrm{P} 123: \mathrm{HCl}: \mathrm{H}_{2} \mathrm{O}$ of $1.0: 0.0163: 6.25: 184$ was employed in this case.

2.3. Drug Loading and In Vitro Release Experiments. Metoprolol was loaded onto the mesoporous matrices through incipient wetness impregnation method. The carriers were added to a $100 \mathrm{gL}^{-1}$ MTP aqueous solution, homogenized, and then dried under vacuum at $25^{\circ} \mathrm{C}$. MTP-loaded materials with 20\% (denoted MTP@“Carrier”) and 50\% (MTP50\%@AlSBA-15) drug content were prepared. The in vitro release experiments were performed in phosphate buffer solution (PBS), $\mathrm{pH}=7.4$, at $37^{\circ} \mathrm{C}$ and constant magnetic stirring rate of $150 \mathrm{rpm}$. A quantity of drug-loaded samples containing $10 \mathrm{mg}$ MTP was added to a dialysis bag (cellulose tubing with average molecular weight cutoff $12000 \mathrm{Da}$, Sigma-Aldrich) together with $1 \mathrm{~mL}$ PBS, sealed, and then immersed in $90 \mathrm{~mL}$ release medium. An in situ UV-Vis probe was used to record the drug concentration every $15 \mathrm{~s}$.

2.4. Characterization. Small- and wide-angle X-ray diffraction (XRD) was performed on a Bruker D8 Discover diffractometer with $\mathrm{Cu} \mathrm{K} \alpha$ radiation $(\lambda=1.5406 \AA)$. Infrared spectroscopy (FT-IR) was carried out on a Bruker Tensor 27 spectrometer (KBr pellets). Scanning electron microscopy (SEM) was recorded on a Tescan Vega 3 LM microscope equipped with an energy dispersive X-ray spectrometer (EDX). Nitrogen adsorption-desorption isotherms were acquired on a Quantachrome Autosorb $\mathrm{iQ}_{2}$ pore analyzer, at $77 \mathrm{~K}$. The samples specific surface area was computed using the Brunauer-Emmett-Teller (BET) theory, in the 0.1-0.3 relative pressure range. The pore size distribution and average pore sizes were calculated with the Barrett-Joyner-Halenda (BJH) model, from both the adsorption and desorption branches of the isotherm. Total pore volume volumes were computed at a relative pressure of 0.80 , which corresponds to mesopores with diameters less than $11 \mathrm{~nm}$. UV-Vis spectroscopy was carried out using an Ocean Optics USB 4000 spectrometer, equipped with an in situ probe. Elemental analysis of the samples was carried out using a wavelength dispersive X-ray fluorescence (WDXRF) spectroscopy method, on a Rigaku ZSX Primus II spectrometer equipped with an Xray tube with $\mathrm{Rh}$ anode, $4.0 \mathrm{~kW}$ power, with front Be window (30 $\mu \mathrm{m}$ thickness). The WDXRF measurements were performed on $10 \mathrm{~mm}$ diameter pressed pellets, under vacuum.

\section{Results and Discussion}

3.1. Characterization of Mesoporous Carriers and MTPLoaded Samples. Both the pristine carriers and drug-loaded samples were characterized in order to determine the relevant properties affecting the drug release process.

Small-angle XRD was used to evidence the ordered mesopore array for both pristine carriers and drug-loaded samples (Figure 1). Three well-defined Bragg reflections for pristine carriers can easily be noticed. These peaks were indexed as the (100), (110), and (200) reflections belonging to the hexagonal $\mathrm{P} 6 \mathrm{~m}$ symmetry group (Figures 1 (a) and 1(b)). The ordered mesopore array is preserved after the introduction of MTP molecules. The presence of the drug molecules inside the carriers mesopores leads to a reduction in the relative intensities of the (110) and (200) reflections, which can be explained by a decrease in the electronic density difference between the silica pore walls and mesopores [39]. 


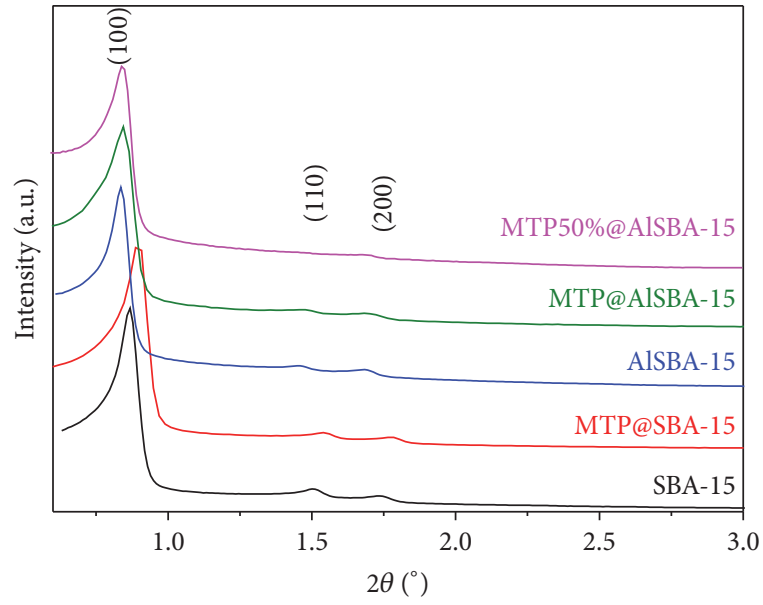

(a)

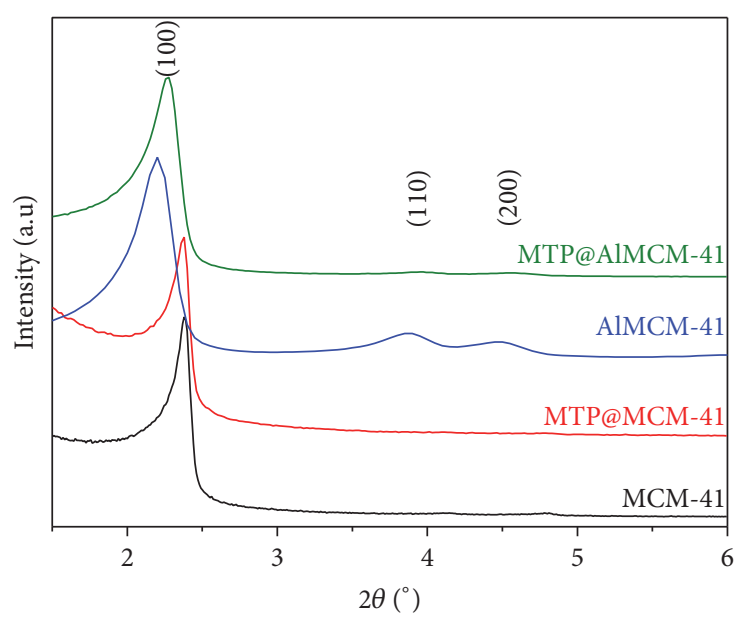

(b)

FIGURE 1: Small-angle XRD patterns for SBA-15-type (a) and MCM-41-type (b) mesoporous carriers and drug-loaded samples.

TABLE 1: Si : Al ratio, drug content, and textural parameters of the samples.

\begin{tabular}{|c|c|c|c|c|c|c|}
\hline \multirow[t]{2}{*}{ Sample } & \multirow{2}{*}{$\mathrm{Si}: \mathrm{Al}$ (at.) } & \multirow{2}{*}{ MTP (\%) } & \multirow{2}{*}{$S_{\mathrm{BET}}\left(\mathrm{m}^{2} \mathrm{~g}^{-1}\right)$} & \multicolumn{2}{|c|}{$d_{\mathrm{BJH}}(\mathrm{nm})$} & \multirow{2}{*}{$V_{\text {pore }}\left(\mathrm{cm}^{3} \mathrm{~g}^{-1}\right)$} \\
\hline & & & & Ads. & Des. & \\
\hline MCM-41 & NA & & 775 & 2.4 & 2.4 & 0.61 \\
\hline AlMCM-41 & 28 & & 1000 & 2.6 & 2.6 & 0.75 \\
\hline SBA-15 & NA & & 870 & 9.6 & 6.6 & 1.16 \\
\hline AlSBA-15 & 116 & & 960 & 9.0 & 6.8 & 1.22 \\
\hline MTP@MCM-41 & $\mathrm{NA}$ & 20 & 79 & NA & NA & 0.08 \\
\hline MTP@AlMCM-41 & 28 & 20 & 726 & 2.1 & 2.0 & 0.34 \\
\hline MTP@SBA-15 & NA & 20 & 334 & 8.1 & 6.3 & 0.61 \\
\hline MTP@AlSBA-15 & 116 & 20 & 398 & 8.1 & 6.3 & 0.68 \\
\hline MTP50\%@AlSBA-15 & 116 & 50 & 111 & 6.8 & 5.1 & 0.19 \\
\hline
\end{tabular}

NA: not applicable/could not be determined.

This effect is more pronounced for AlSBA-15 materials, which exhibit a gradual decrease in the secondary peak intensity with increasing drug content. The wide-angle XRD patterns of MTP-loaded samples (Figure 2(a)) show no diffraction peaks, indicating that the drug molecules are present only in the carrier mesopores, in amorphous state.

The FT-IR spectra (Figure 2(b)) of both pristine carriers and MTP-loaded samples show the characteristic vibrations of the aluminosilicate matrix: $\mathrm{Si}-\mathrm{O}-\mathrm{Si}$ symmetric and asymmetric stretching superimposed with the corresponding $\mathrm{Si}-\mathrm{O}-\mathrm{Al}$ vibrations $\left(800 \mathrm{~cm}^{-1}, 1090 \mathrm{~cm}^{-1}\right)$, Si-O bending $\left(460 \mathrm{~cm}^{-1}\right)$, Al-O bending $\left(570 \mathrm{~cm}^{-1}\right)$, and Si-OH stretching vibration $\left(960 \mathrm{~cm}^{-1}\right)$. The presence of hydroxyl stretching vibration (broad band, $3400 \mathrm{~cm}^{-1}$ ) and adsorbed water $\left(1640 \mathrm{~cm}^{-1}\right)$ can also be noticed in all samples. The presence of the drug molecules for the MTP-loaded materials can be evidenced through the MTP characteristic vibrations, such as C-H stretching $\left(2850-2990 \mathrm{~cm}^{-1}\right)$ and the $\mathrm{C}-\mathrm{H}, \mathrm{C}=\mathrm{C}$ vibrations in the "fingerprint" region (1550-1400 $\mathrm{cm}^{-1}$ ).

SEM analysis was carried out to assess the morphology of the mesoporous carriers (Figure 3). The SBA-15-type supports
(Figures 3(a) and 3(b)) present a characteristic rod-like morphology, while the aluminum-doped MCM-41 sample consists of irregular particles (Figure 3(c)). The elemental distribution was evaluated using EDX spectroscopy. The aluminosilicate carriers show a homogenous distribution of aluminum into the silica framework (Figures 3(d), 3(e), and 3(f)). WDXRF measurements were performed in order to quantitatively assess the $\mathrm{Si}: \mathrm{Al}$ ratio. $\mathrm{Si}$ : Al ratio of $1: 28$ was obtained for the commercial AlMCM-41, while the prepared AlSBA-15 carrier has a 1:116 Si : Al ratio (Table 1).

The nitrogen adsorption-desorption isotherms of the carriers and AlSBA-15 drug-loaded materials are of type IV with hysteresis, characteristic for mesoporous samples (Figure 4). The adsorbed gas volume decreases with increasing drug content in the case of AlSBA-15 samples, demonstrating that the drug molecules are adsorbed inside the support mesochannels (Figure 4(A, B, and C)). A similar decrease can be also noticed for MCM-41-type materials after MTP loading (Figure 4(D and E)). The average pore size decreases after drug loading, indicating that there are strong interactions between the biologically active substance and the 


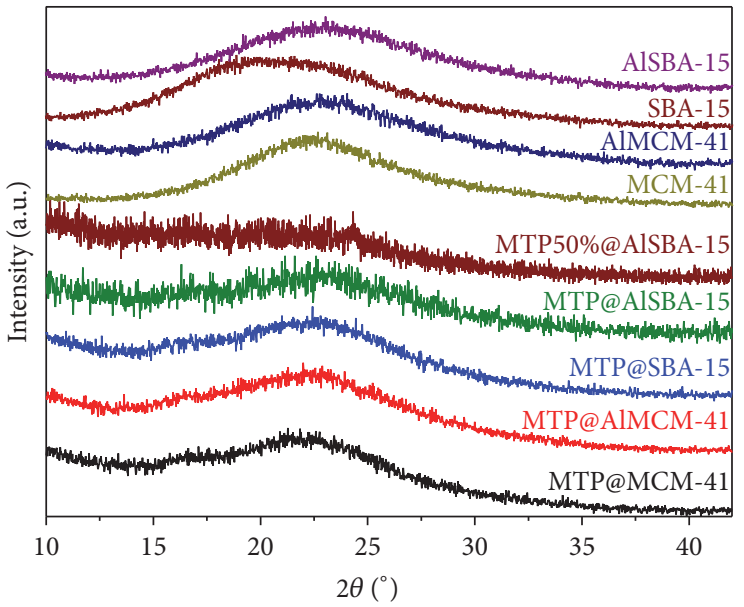

(a)

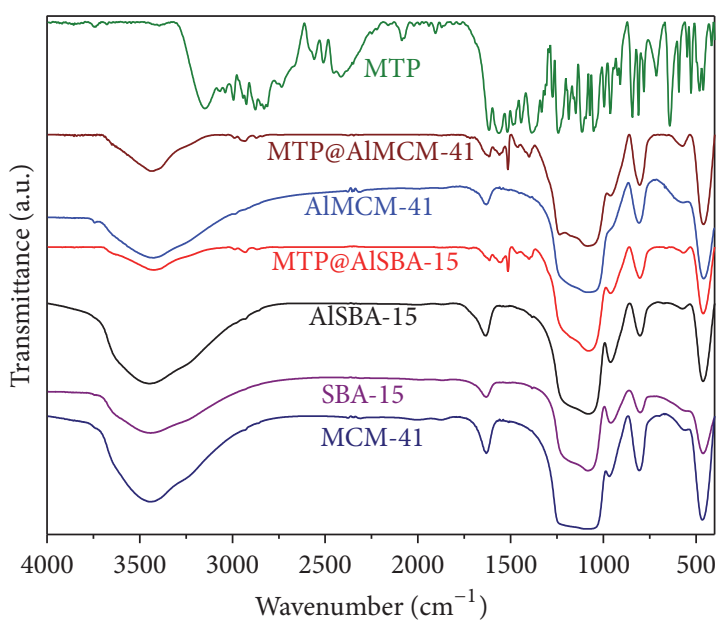

(b)

FIGURE 2: Wide-angle XRD patterns of carriers and drug-loaded samples (a) and FT-IR spectra of mesoporous carriers, drug-loaded materials, and MTP (b).

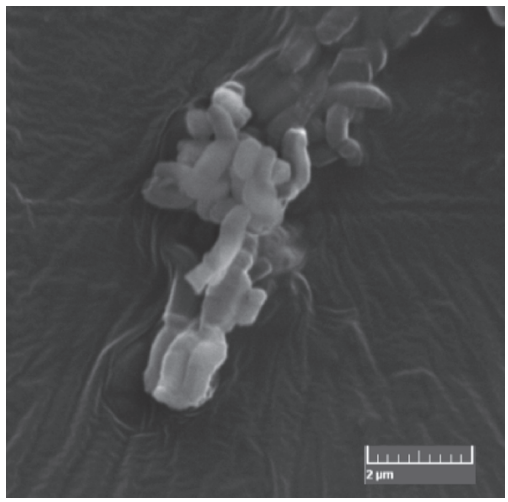

(a)

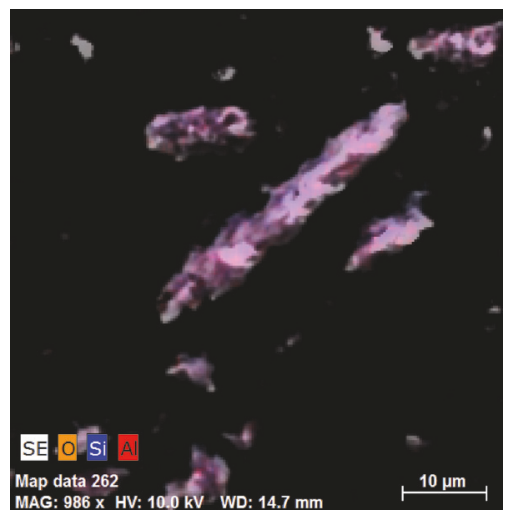

(d)

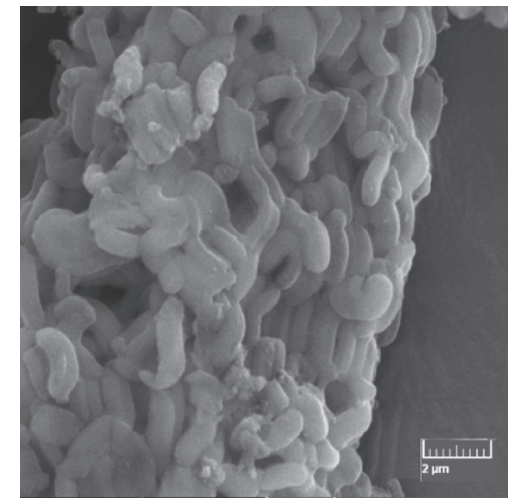

(b)

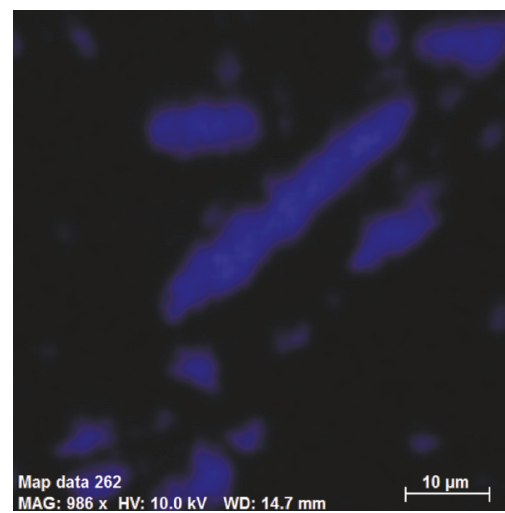

(e)

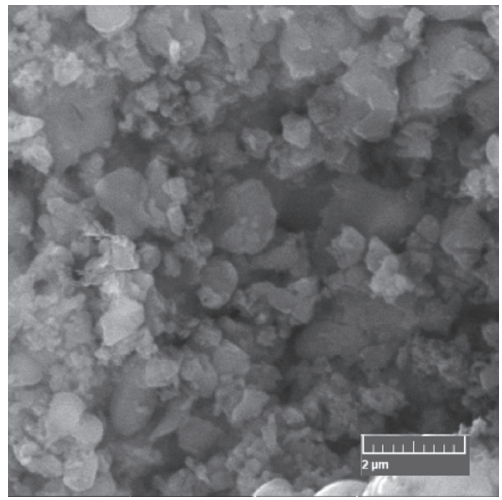

(c)

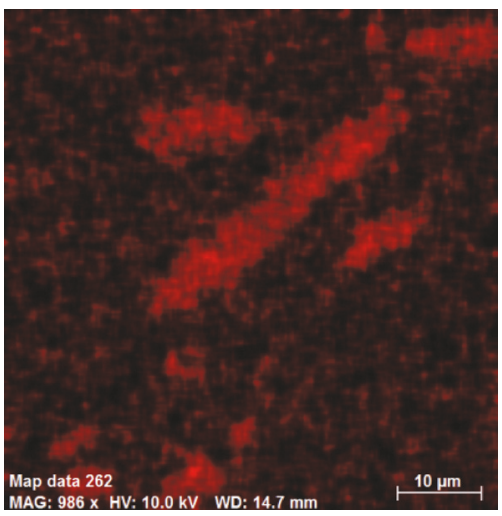

(f)

FIGURE 3: SEM analysis of SBA-15 (a), AlSBA-15 (b), and AlMCM-41 supports (c), AlSBA15 EDX elemental mapping (d), and distribution of $\mathrm{Si}(\mathrm{e})$ and $\mathrm{Al}(\mathrm{f})$.

aluminosilicate pore walls. Similarly, the specific surface area and total pore volume decrease after MTP loading (Table 1).

3.2. In Vitro Drug Delivery. The metoprolol release profiles were obtained in phosphate buffer solution, $\mathrm{pH}=7.4,37^{\circ} \mathrm{C}$ for all samples, using the dialysis bag method and compared with the drug diffusion in similar conditions (Figure 5). The MTP diffusion through the dialysis membrane is relatively fast, reaching $90 \%$ cumulative release after $2.5 \mathrm{~h}$. In contrast, the drug release kinetics from the mesoporous carriers is 

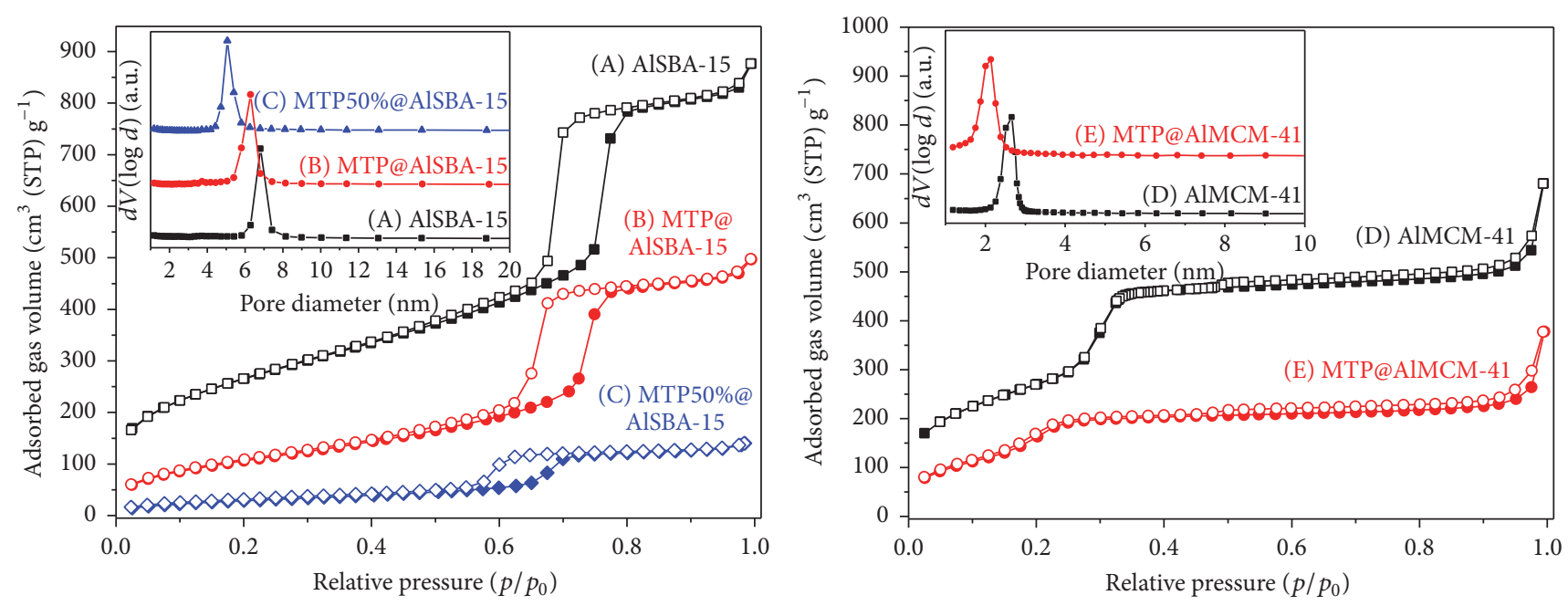

FIGURE 4: Nitrogen adsorption-desorption isotherms of mesoporous carriers and drug-loaded samples. Inset: the corresponding pore size distribution curves.

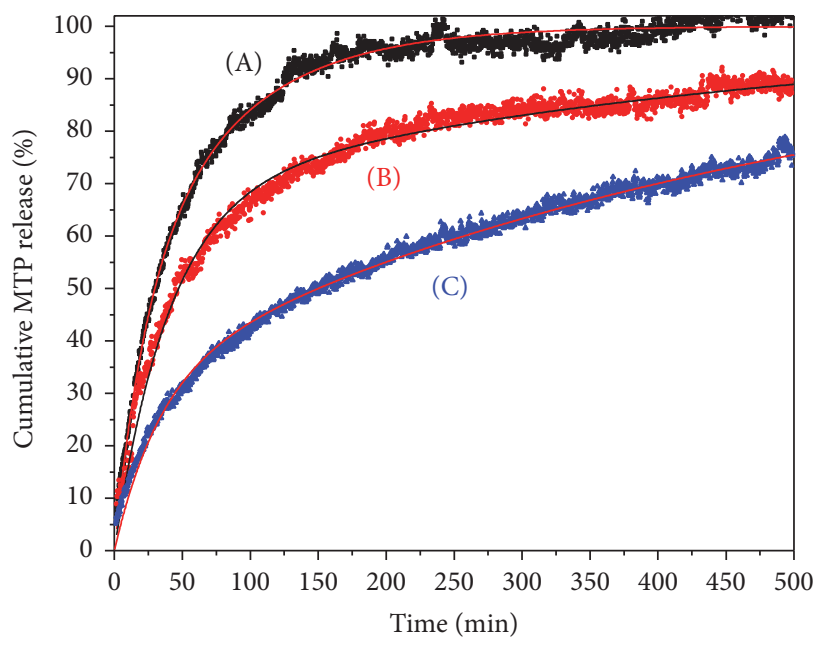

- (A) MTP

- (B) MTP@MCM-41

•(C) MTP@AlMCM-41

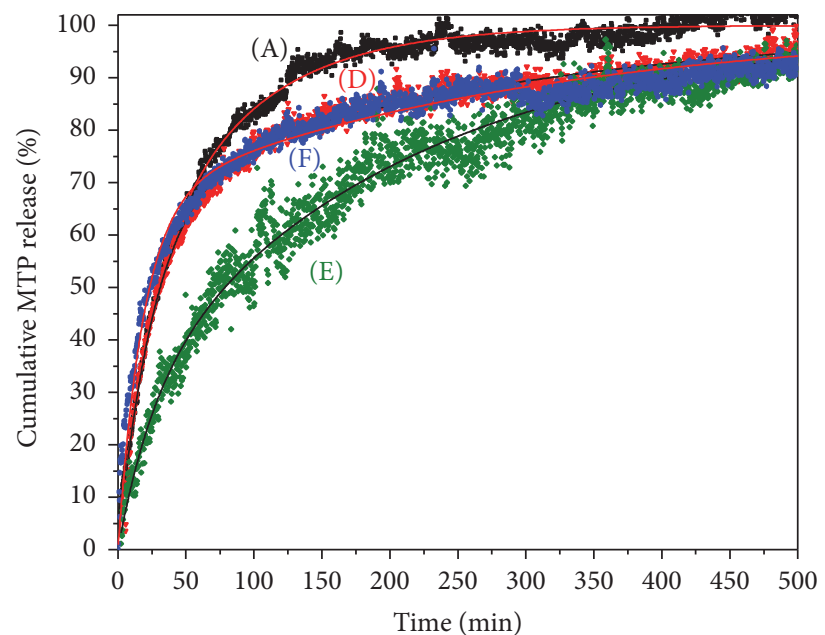

- (A) MTP

- (D) MTP@SBA-15

- (E) MTP@AlSBA-15

-(F) MTP50\%@AlSBA-15

FIGURE 5: Cumulative MTP release profiles, obtained using the dialysis bag method. Symbols denote experimental data, while continuous lines represent fitted data.

slower, signifying the fact that these mesoporous materials can control the drug delivery. The presence of Al leads to marked decrease MTP release rate in the case of MCM41-type samples (Figure 5(B) versus 5(C)). The same effect can be noticed for SBA-15 and AlSBA-15 matrices loaded with $20 \%$ wt. biologically active substance (Figure 5(D) versus $5(\mathrm{E})$ ). Interestingly, increasing the drug loading in AlSBA-15 to $50 \%$ leads to faster release rates, comparable to that of 20\% MTP loaded in SBA-15 (Figure 5(D) versus $5(\mathrm{~F})$ ). This effect could be understood as arising from the reduced supramolecular interactions between drug molecule and the aluminosilicate pore walls, as only a fraction of MTP molecules in MTP50\%@AlSBA-15 can be adsorbed directly onto the mesopore surface. A comparison of MTP-loaded
MCM-41 and SBA-15 materials shows that samples containing mesoporous carriers with larger-pore sizes exhibit faster drug release kinetics than the materials with smaller pore diameters (Figure 5(B) versus 5(D)), in line with previous data [31]. The same observation can be made for samples containing commercial AlMCM-41 and prepared AlSBA-15 supports (Figure $5(\mathrm{C}$ ) versus $5(\mathrm{E})$ ), even though in this case the difference in release rate could also be explained by the higher Al content of AlMCM-41 carrier (Fig. S1).

In order to gain additional insight regarding the MTP release process, the experimental data was fitted with a threeparameter kinetic model developed by Zeng et al. (Figure 5, continuous lines) [29]. This model considers that the drug release consists of three processes, each assumed to follow 
TABLE 2: Kinetics release parameters for the MTP-loaded samples.

\begin{tabular}{lcccr}
\hline Sample & $k_{d}\left(10^{3} \mathrm{~min}^{-1}\right)$ & $k_{\text {off }}\left(10^{3} \mathrm{~min}^{-1}\right)$ & $k_{\text {on }}\left(10^{3} \mathrm{~min}^{-1}\right)$ & $\Delta G\left(10^{-21} \mathrm{~J}\right)$ \\
\hline MTP & 35.6 & 16.0 & 5.4 & 4.65 \\
MTP@MCM-41 & 24.0 & 2.2 & 0.8 & 4.31 \\
MTP@AlMCM-41 & 26.8 & 2.3 & 3.1 & 0.9475 \\
MTP@SBA-15 & 35.4 & 3.8 & 1.3 & 0.9881 \\
MTP@AlSBA-15 & 29.5 & 6.1 & 6.5 & 0.9951 \\
MTP50\%@AlSBA-15 & 51.9 & 3.6 & 1.5 & 0.99 \\
\hline
\end{tabular}

1st-order kinetics. Initially, the drug molecules can either be adsorbed onto the carrier surface (termed "associated") or dissociated from the surface, into the silica mesopores. The association and dissociation processes are reversible and follow 1st-order kinetics, with the $k_{\text {on }}$ and $k_{\text {off }}$ rate constants, respectively. The molecular Gibbs energy of the associationdissociation process can be computed as

$$
\Delta G=-k_{B} T \cdot \ln \left(\frac{k_{\text {on }}}{k_{\text {off }}}\right),
$$

where $k_{B}$ is Boltzmann's constant and $T$ is the temperature.

The dissociated molecules are then transported into the release medium through diffusion, also approximated as a 1storder process, with the rate constant $k_{d}$. It is worth noting that the transport process includes the contributions of diffusion through mesopores and the dialysis membrane. The analytical function corresponding to the theoretical model is presented in

$$
\begin{aligned}
\frac{m(t)}{m(0)}= & \frac{\lambda_{2}\left(k_{d}-\lambda_{2}\right)}{\left(k_{\text {on }}+k_{\text {off }}\right)\left(\lambda_{1}-\lambda_{2}\right)}\left(1-e^{-\lambda_{1} t}\right) \\
& +\frac{\lambda_{1}\left(\lambda_{1}-k_{d}\right)}{\left(k_{\text {on }}+k_{\text {off }}\right)\left(\lambda_{1}-\lambda_{2}\right)}\left(1-e^{-\lambda_{2} t}\right),
\end{aligned}
$$

where $m(0)$ and $m(t)$ are the initial drug content and the cumulative MTP amount released at time $t, k_{\text {on }}, k_{\text {off }}$, and $k_{d}$ are the 1st-order rate constants, and

$$
=\frac{\left[\left(k_{d}+k_{\text {on }}+k_{\text {off }}\right) \pm \sqrt{\left(k_{d}+k_{\text {on }}+k_{\text {off }}\right)^{2}-4 k_{s} k_{\text {off }}}\right]}{2} .
$$

The MTP release is a two-stage process, consisting of a fast, initial release stage ("burst stage"), which is followed by a more gradual drug release, in the sustained release step. The kinetic parameters of the theoretical model can be used to characterize the drug release process. The rate constant for the diffusion process, $k_{d}$, is proportional to the drug release rate during the burst release stage. Since dissociation is slower than diffusion, the initially dissociated MTP molecules are released in this phase and thus the kinetics of the burst release are determined by the rate of diffusion for the initially dissociated molecules. The dissociation rate constant, $k_{\text {off }}$, is proportional to the drug release rate during the sustained release regime, as most initially dissociated molecules have been released in the burst phase and dissociation becomes the rate limiting step. Finally, the MTP percentage released during the burst stage is proportional to the Gibbs energy, $\Delta G$, which drives the equilibrium between association and dissociation and therefore the amount of initially dissociated molecules.

The values of the three rate constants, $k_{d}, k_{\text {off }}$, and $k_{\text {on }}$, have been determined for each drug-loaded sample and MTP by fitting the experimental release profiles with (2) (Table 2). The value of the association-dissociation Gibbs energy was then computed using (1). All MTP-silica samples show a good correlation between the experimental and fitted data, with $R^{2}>0.96$. MTP dissolution was fitted with the same model, purely for comparison reasons, as this case could also be explained by a simple first-order kinetics model.

The $\Delta G$ parameter provides information regarding the amount of drug released during the burst phase of the release profile. Lower $\Delta G$ values indicate that a smaller drug percentage is released during the burst regime, as the association-dissociation equilibrium is shifted towards association. Doping the mesoporous silica matrix with Al has a clear effect on the Gibbs energy values of the MTP-loaded samples (Table 2). Both MTP@AlMCM-41 and MTP@AlSBA-15 have negative $\Delta G$, while samples containing pristine silica present positive $\Delta G$ values. This effect can be easily understood as the introduction of $\mathrm{Al}$ atoms in the silica matrix generates negative charges, which yield stronger electrostatic interactions with the positively charged drug molecules than in the case of silica (silanol groups are also negatively charged at $\mathrm{pH}$ 7.4). It is interesting to note that increasing the loaded MTP amount to $50 \%$ wt. leads to an increase in $\Delta G$ (MTP50\%@AlSBA-15 versus MTP@AlSBA15). This observation supports the electrostatic interaction interpretation, as only a fraction of MTP molecules can be adsorbed on the pore surface, irrespective of drug loading. A higher drug content shifts the association-dissociation equilibrium towards dissociation as the mesopore surface becomes saturated with associated MTP molecules and the excess drug can only be accommodated as dissociated species (i.e., without interactions with the aluminosilicate surface).

The release rate during the burst stage is proportional with the diffusion rate constant, $k_{d}$, with higher rate constant values indicating faster drug release. The $k_{d}$ values are similar for all $20 \%$ wt. MTP-loaded samples, with slightly lower values for MCM-41-type samples (MTP@MCM-41 and MTP@AlMCM-41) than for the SBA-15-type materials. The drug transport process through the matrix mesopores is not affected by the presence or absence of $\mathrm{Al}$, but it is slightly influenced by the pore size. As expected, the larger pores 
TABLE 3: Correlation coefficients between release kinetics parameters and sample textural parameters. Bold values indicate absolute correlation values greater than \pm 0.75 .

\begin{tabular}{lcccc}
\hline Parameters & $k_{d}$ & $k_{\text {off }}$ & $k_{\text {on }}$ & $\Delta G$ \\
\hline $\mathrm{Al} / \mathrm{Si}$ & -0.18 & -0.27 & 0.30 & $-\mathbf{0 . 8 2}$ \\
$S_{\text {carrier }}$ & 0.33 & 0.33 & 0.57 & -0.73 \\
$d_{\text {carrier }}$ & 0.67 & $\mathbf{0 . 7 9}$ & 0.29 & 0.21 \\
$V_{\text {carrier }}$ & 0.69 & $\mathbf{0 . 7 9}$ & 0.36 & 0.08 \\
$S_{\text {loaded }}$ & -0.39 & 0.00 & 0.48 & $-\mathbf{0 . 8 4}$ \\
$d_{\text {loaded }}$ & 0.50 & $\mathbf{0 . 8 3}$ & 0.37 & 0.14 \\
$V_{\text {loaded }}$ & -0.10 & $\mathbf{0 . 7 7}$ & 0.66 & -0.34 \\
$S_{\text {drug }}{ }^{*}$ & 0.49 & 0.12 & -0.37 & $\mathbf{0 . 7 7}$ \\
$\Delta d_{\text {drug }}$ & $\mathbf{0 . 7 7}$ & -0.12 & -0.31 & 0.30 \\
$V_{\text {drug }}{ }^{* *}$ & $\mathbf{0 . 8 3}$ & -0.10 & -0.44 & 0.54 \\
\hline
\end{tabular}

${ }^{*}$ Equation (4) and ${ }^{* *}$ equation (5).

of SBA-15-type materials lead to a faster transport than the smaller MCM-41 pores. Increasing the drug content to $50 \%$ leads to a large increase in $k_{d}$ and in the release rate during the burst stage. This fact suggests that a larger fraction of MTP molecules are adsorbed on the external particle surface or near the pore entrances, resulting in shorter diffusion paths than for the $20 \%$ drug-loaded sample.

The rate of therapeutic agent release during the sustained release stage is directly proportional to the dissociation rate constant, $k_{\text {off }}$. MCM-41-type samples show significantly smaller $k_{\text {off }}$ values than the SBA-15 samples. The Al insertion into the silica framework does not influence the dissociation rate constant for MCM-41 samples, while increasing its value in the case of SBA-15-type materials. Moreover, the variation of $k_{\text {off }}$ (MCM-41 < AlMCM-41 < SBA-15 < AlSBA-15) is similar to the variation of total pore volume (Table 1 ). The increase in dissociation rate could be explained by steric effects. The larger SBA-15 mesopores $(6 \mathrm{~nm})$ favor the drug desorption as there is less steric hindrance of the dissociated molecules than in the case of the smaller MCM-41 mesopores $(2.5 \mathrm{~nm})$. The MTP molecule has an estimated length of $1.6 \mathrm{~nm}$, which is approximately $60 \%$ of the MCM-41 pore diameter but only $25 \%$ of the SBA- 15 pore size. The influence of steric crowding on the dissociation rate can also be noticed when comparing the $20 \%$ and 50\% wt. drug-loaded AlSBA- 15 samples, with the higher drug loading resulting in a decreased $k_{\text {off }}$ parameter (Table 2).

A correlation analysis between the kinetics release parameters $\left(k_{d}, k_{\text {on }}, k_{\text {off }}\right.$, and $\left.\Delta G\right)$ and various textural parameters for carriers and drug-loaded samples has been carried out (Table 3$)$. The specific surface area $(S)$, average pore diameter $(d)$, and total pore volume $(V)$ of both pristine mesoporous carriers (index "carrier") and drug-loaded samples (index "loaded") have been considered (Table 3). Other parameters such as the $\mathrm{Al} / \mathrm{Si}$ atomic ratio, the total pore volume occupied by the MTP molecules $\left(V_{\text {drug }}(4)\right)$, the specific surface area decrease after drug adsorption $\left(S_{\text {drug }}(5)\right)$, and the difference between the average pore size of the pristine carrier and drug-loaded samples $\left(\Delta d_{\mathrm{drug}}=d_{\mathrm{BJH}}\right.$, carrier $-d_{\mathrm{BJH}}$, drugloaded sample) have been computed and included in Table 3.
The correlation results in Table 3 take values between -1 and 1 , with values close to \pm 1 indicating positive or negative correlation and values close to 0 indicating no correlation between the two parameters.

$$
S_{\text {drug }}=S_{\text {carrier }}-S_{\text {loaded }} \cdot \frac{100}{(100-\% \mathrm{MTP})},
$$

where $S_{\text {drug }}, S_{\text {carrier }}$, and $S_{\text {loaded }}$ represent the specific surface area decrease after drug adsorption, the specific surface area of the pristine carrier, and the drug-loaded sample, respectively.

$$
V_{\text {drug }}=V_{\text {carrier }}-V_{\text {loaded }} \cdot \frac{100}{(100-\% \mathrm{MTP})},
$$

where $V_{\text {drug }}, V_{\text {carrier }}$, and $V_{\text {loaded }}$ represent the volume occupied by the drug molecules and the total pore volumes of the pristine carrier and of the drug-loaded sample, respectively, and \% MTP represents the drug loading percentage.

The correlation results support the qualitative observations regarding the influence of textural properties of the carriers and MTP-loaded samples on the kinetic parameters. The $\mathrm{Al} / \mathrm{Si}$ ratio has a strong negative correlation with the $\Delta G$ parameter, signifying that the Gibbs energy decreases with increasing $\mathrm{Al}$ content. The $\mathrm{Al} / \mathrm{Si}$ ratio is not correlated with any other kinetic parameters, suggesting that $\mathrm{Al}$ doping is a promising approach to tailor the ratio of drug released in the burst to sustained release stages, without affecting the release rates. The $\Delta G$ parameter is also inversely correlated with the specific surface area of the drug-loaded samples and it is directly correlated with the specific surface area decrease after drug adsorption, suggesting the importance of the surfacedrug interactions in the association-dissociation equilibrium.

The diffusion rate constant, $k_{d}$, responsible for the release rate during the burst stage is directly correlated with the pore volume occupied by the MTP molecules $\left(V_{\text {drug }}\right)$ and the volume pore size difference between the pristine carrier and drug-loaded sample $\left(V_{\text {drug }}, \Delta d_{\text {drug }}\right)$. The correlation between the volume occupied by drug molecules and the diffusion rate indicates that increasing the therapeutic agent content leads to a greater fraction of molecules which can be readily transported in the release medium and thus to faster diffusion rates.

The dissociation rate constant, $k_{\text {off }}$, which influences the drug release rate during the sustained release stage, is correlated with the pore size and pore volume of both pristine carriers and drug-loaded samples. These correlations support the hypothesis that dissociation rates are mainly influenced by the steric crowding of the drug molecules in the dissociated state.

\section{Conclusions}

In this work the possibility of tailoring the drug release kinetics of a model drug, metoprolol, from mesoporous silica and aluminosilicate materials was studied. Two types of mesoporous carriers with hexagonal ordered pore arrays, MCM-41 and SBA-15, were employed. The experimental metoprolol release kinetics were fitted with a three-parameter 
kinetic model, which consists of an equilibrium between drug adsorption and desorption onto the mesopore surface, followed by transport into the release medium. The influence of doping the silica framework with aluminum was studied by correlating the kinetic parameters of the drug release process with the carriers textural properties.

It was found that all mesoporous carriers could act as drug reservoirs. The larger-pore SBA-15-type carriers could be loaded with up to $50 \% \mathrm{wt}$. biologically active molecules. All drug release profiles consist of a two-stage process, with a fast initial drug release in the burst stage, followed by a more gradual release, in the sustained release regime. The whole drug release process is characterized by three kinetic parameters, namely, the metoprolol release rates during the burst and sustained release stages, respectively, and the Gibbs energy of the adsorption/desorption equilibrium. These three parameters are proportional to the rate constant of the transport process, the rate of drug desorption from the pore surface, and the ratio between the amounts of therapeutic agent released in the burst and sustained release stages, respectively.

The drug release rate during the burst stage is directly correlated with the drug-loaded amount and the average pore size of the carriers. The sustained release rate was found to be correlated with the steric crowding of the dissociated molecules inside the support mesopores, with a higher carrier pore volume resulting in faster release rate.

The introduction of $\mathrm{Al}$ atoms into the silica framework leads to the decrease of Gibbs energy and consequently of the drug amount released in the burst stage, while not affecting the other kinetic parameters. This effect is explained by the electrostatic interactions arising between the negatively charged pore surface and the positively charged model drug molecules and it also depends on the amount of therapeutic agent loaded into the mesoporous carriers. A higher drug loading leads to a decrease in average electrostatic interaction strength and higher overall Gibbs energy. Doping the silica framework with heteroatoms is therefore a promising strategy towards the rational design of drug release systems which combine both instantaneous and sustained release. In prospective, the heteroatom doping of mesoporous silica could be combined with varying the drug amount and carriers textural properties in order to precisely tailor the release profiles of advanced drug delivery systems.

\section{Conflicts of Interest}

The authors declare that they have no conflicts of interest.

\section{Acknowledgments}

The authors are grateful for the financial support of the Romanian Project PCCA no. 131/2012.

\section{References}

[1] F. Tang, L. Li, and D. Chen, "Mesoporous silica nanoparticles: synthesis, biocompatibility and drug delivery," Advanced Materials, vol. 24, no. 12, pp. 1504-1534, 2012.
[2] Z. Li, J. C. Barnes, A. Bosoy, J. F. Stoddart, and J. I. Zink, "Mesoporous silica nanoparticles in biomedical applications," Chemical Society Reviews, vol. 41, no. 7, pp. 2590-2605, 2012.

[3] M. Vallet-Regí, F. Balas, and D. Arcos, "Mesoporous materials for drug delivery," Angewandte Chemie, vol. 46, no. 40, pp. 7548-7558, 2007.

[4] C. Charnay, S. Bégu, C. Tourné-Péteilh, L. Nicole, D. A. Lerner, and J. M. Devoisselle, "Inclusion of ibuprofen in mesoporous templated silica: drug loading and release property," European Journal of Pharmaceutics and Biopharmaceutics, vol. 57, no. 3, pp. 533-540, 2004.

[5] S. A. Jadhav, V. Brunella, G. Berlier, E. Ugazio, and D. Scalarone, "Effect of multimodal pore channels on cargo release from mesoporous silica nanoparticles," Journal of Nanomaterials, vol. 2016, Article ID 1325174, 2016.

[6] A. L. Doadrio, A. J. Salinas, J. M. Sánchez-Montero, and M. Vallet-Regí, "Drug release from ordered mesoporous silicas," Current pharmaceutical design, vol. 21, no. 42, pp. 6213-6819, 2015.

[7] S. K. Natarajan and S. Selvaraj, "Mesoporous silica nanoparticles: importance of surface modifications and its role in drug delivery," RSC Advances, vol. 4, no. 28, pp. 14328-14334, 2014.

[8] V. Nairi, L. Medda, M. Monduzzi, and A. Salis, "Adsorption and release of ampicillin antibiotic from ordered mesoporous silica," Journal of Colloid and Interface Science, vol. 497, pp. 217-225, 2017.

[9] L. Pasqua, L. Veltri, B. Gabriele, F. Testa, and G. Salerno, "Progesterone inclusion into cyclodextrin-functionalized mesoporous silica," Journal of Porous Materials, vol. 20, no. 4, pp. 917925, 2013.

[10] X. Feng, G. E. Fryxell, L.-Q. Wang, A. Y. Kim, J. Liu, and K. M. Kemner, "Functionalized monolayers on ordered mesoporous supports," Science, vol. 276, no. 5314, pp. 923-926, 1997.

[11] F. Sevimli and A. Yilmaz, "Surface functionalization of SBA-15 particles for amoxicillin delivery," Microporous and Mesoporous Materials, vol. 158, pp. 281-291, 2012.

[12] J. D. Webb, T. Seki, J. F. Goldston, M. Pruski, and C. M. Crudden, "Selective functionalization of the mesopores of SBA15," Microporous and Mesoporous Materials, vol. 203, no. C, pp. 123-131, 2015.

[13] S. Iqbal and J. Yun, "EDTA-functionalized mesoporous silica for the removal of corrosion products: Adsorption studies and performance evaluation under gamma irradiation," Microporous and Mesoporous Materials, vol. 248, pp. 149-157, 2017.

[14] D. Berger, S. Nastase, R. A. Mitran et al., "Mesostructured silica and aluminosilicate carriers for oxytetracycline delivery systems," International Journal of Pharmaceutics, vol. 510, no. 2, pp. 524-531, 2016.

[15] R. B. Borade and A. Clearfield, "Synthesis of aluminum rich MCM-41," Catalysis Letters, vol. 31, no. 2-3, pp. 267-272, 1995.

[16] D. Q. Khieu, D. T. Quang, T. D. Lam, N. H. Phu, J. H. Lee, and J. S. Kim, "Fe-MCM-41 with highly ordered mesoporous structure and high Fe content: synthesis and application in heterogeneous catalytic wet oxidation of phenol," Journal of Inclusion Phenomena and Macrocyclic Chemistry, vol. 65, no. 1, pp. 73-81, 2009.

[17] A. Ranoux, K. Djanashvili, I. W. C. E. Arends, and U. Hanefeld, "B-TUD-1: A versatile mesoporous catalyst," RSC Advances, vol. 3, no. 44, pp. 21524-21534, 2013.

[18] E. M. Johansson, M. A. Ballem, J. M. Córdoba, and M. Odén, "Rapid synthesis of SBA-15 rods with variable lengths, widths, 
and tunable large pores," Langmuir, vol. 27, no. 8, pp. 49944999, 2011.

[19] N. Ž. Knežević and J.-O. Durand, "Large pore mesoporous silica nanomaterials for application in delivery of biomolecules," Nanoscale, vol. 7, no. 6, pp. 2199-2209, 2015.

[20] T. Ogura, K. Sakai, H. Sakai, and M. Abe, "Synthesis of highly ordered mesoporous silica with a lamellar structure using assembly of cationic and anionic surfactant mixtures as a template," Journal of Physical Chemistry C, vol. 112, no. 32, pp. 12184-12187, 2008.

[21] E. M. Björk, F. Söderlind, and M. Odén, “Tuning the shape of mesoporous silica particles by alterations in parameter space: from rods to platelets," Langmuir, vol. 29, no. 44, pp. 1355113561, 2013.

[22] A. Chang, N.-C. Lai, and C.-M. Yang, "MCM-48 nanorods: a self-assembled isotropic cubic mesostructure with anisotropic morphology," RSC Advances, vol. 2, no. 32, pp. 12088-12090, 2012.

[23] Y. Han, D. Zhang, L. L. Chng et al., "A tri-continuous mesoporous material with a silica pore wall following a hexagonal minimal surface," Nature Chemistry, vol. 1, no. 2, pp. 123-127, 2009.

[24] M. A. Ballem, J. M. Córdoba, and M. Odén, "Influence of synthesis temperature on morphology of SBA-16 mesoporous materials with a three-dimensional pore system," Microporous and Mesoporous Materials, vol. 129, no. 1-2, pp. 106-111, 2010.

[25] S. Nanaki, M. Tseklima, Z. Terzopoulou et al., "Use of mesoporous cellular foam (MCF) in preparation of polymeric microspheres for long acting injectable release formulations of paliperidone antipsychotic drug," European Journal of Pharmaceutics and Biopharmaceutics, vol. 117, pp. 77-90, 2017.

[26] M. Manzano and M. Vallet-Regí, "New developments in ordered mesoporous materials for drug delivery," Journal of Materials Chemistry, vol. 20, no. 27, pp. 5593-5604, 2010.

[27] R.-A. Mitran, S. Nastase, C. Matei, and D. Berger, "Tailoring the dissolution rate enhancement of aminoglutethimide by functionalization of MCM-41 silica: A hydrogen bonding propensity approach," RSC Advances, vol. 5, no. 4, pp. 2592-2601, 2015.

[28] P. T. Wong and S. K. Choi, "Mechanisms of drug release in nanotherapeutic delivery systems," Chemical Reviews, vol. 115, no. 9, pp. 3388-3432, 2015.

[29] L. Zeng, L. An, and X. Wu, "Modeling drug-carrier interaction in the drug release from nanocarriers," Journal of Drug Delivery, vol. 2011, Article ID 370308, 15 pages, 2011.

[30] M. Martínez-Carmona, M. Colilla, M. L. Ruiz-González, J. M. González-Calbet, and M. Vallet-Regí, "High resolution transmission electron microscopy: a key tool to understand drug release from mesoporous matrices," Microporous and Mesoporous Materials, vol. 225, pp. 399-410, 2016.

[31] R. Mitran, C. Matei, and D. Berger, "Correlation of mesoporous silica structural and morphological features with theoretical three-parameter model for drug release kinetics," The Journal of Physical Chemistry C, vol. 120, no. 51, pp. 29202-29209, 2016.

[32] “Register Federal," Vol. 45, No. 13, 1980.

[33] E. Ghedini, M. Signoretto, F. Pinna, V. Crocellà, L. Bertinetti, and G. Cerrato, "Controlled release of metoprolol tartrate from nanoporous silica matrices," Microporous and Mesoporous Materials, vol. 132, no. 1-2, pp. 258-267, 2010.

[34] Z. Guo, Y. Du, X. Liu, S.-C. Ng, Y. Chen, and Y. Yang, "Enantioselectively controlled release of chiral drug (metoprolol) using chiral mesoporous silica materials," Nanotechnology, vol. 21, no. 16, Article ID 165103, 2010.
[35] M. Moritz and M. Łaniecki, "Modified SBA-15 as the carrier for metoprolol and papaverine: adsorption and release study," Journal of Solid State Chemistry, vol. 184, no. 7, pp. 1761-1767, 2011.

[36] S. Nastase, L. Bajenaru, C. Matei, R. A. Mitran, and D. Berger, "Ordered mesoporous silica and aluminosilicate-type matrix for amikacin delivery systems," Microporous and Mesoporous Materials, vol. 182, pp. 32-39, 2013.

[37] S. Lin, L. Shi, M. M. L. Ribeiro Carrott et al., "Direct synthesis without addition of acid of Al-SBA-15 with controllable porosity and high hydrothermal stability," Microporous and Mesoporous Materials, vol. 142, no. 2-3, pp. 526-534, 2011.

[38] R. A. Mitran, S. Nastase, L. Bajenaru, C. Matei, and D. Berger, "Mesostructured aluminosilicates as carriers for doxycyclinebased drug delivery systems," in Proceedings of the 14th SGEM GeoConference on Nano, Bio and Green: Technologies for A Sustainable Future, 120, 113 pages, 2014.

[39] M. Imperor-Clerc, P. Davidson, and A. Davidson, "Existence of a microporous corona around the mesopores of silica-based SBA-15 materials templated by triblock copolymers," Journal of the American Chemical Society, vol. 122, no. 48, pp. 11925-11933, 2000 . 

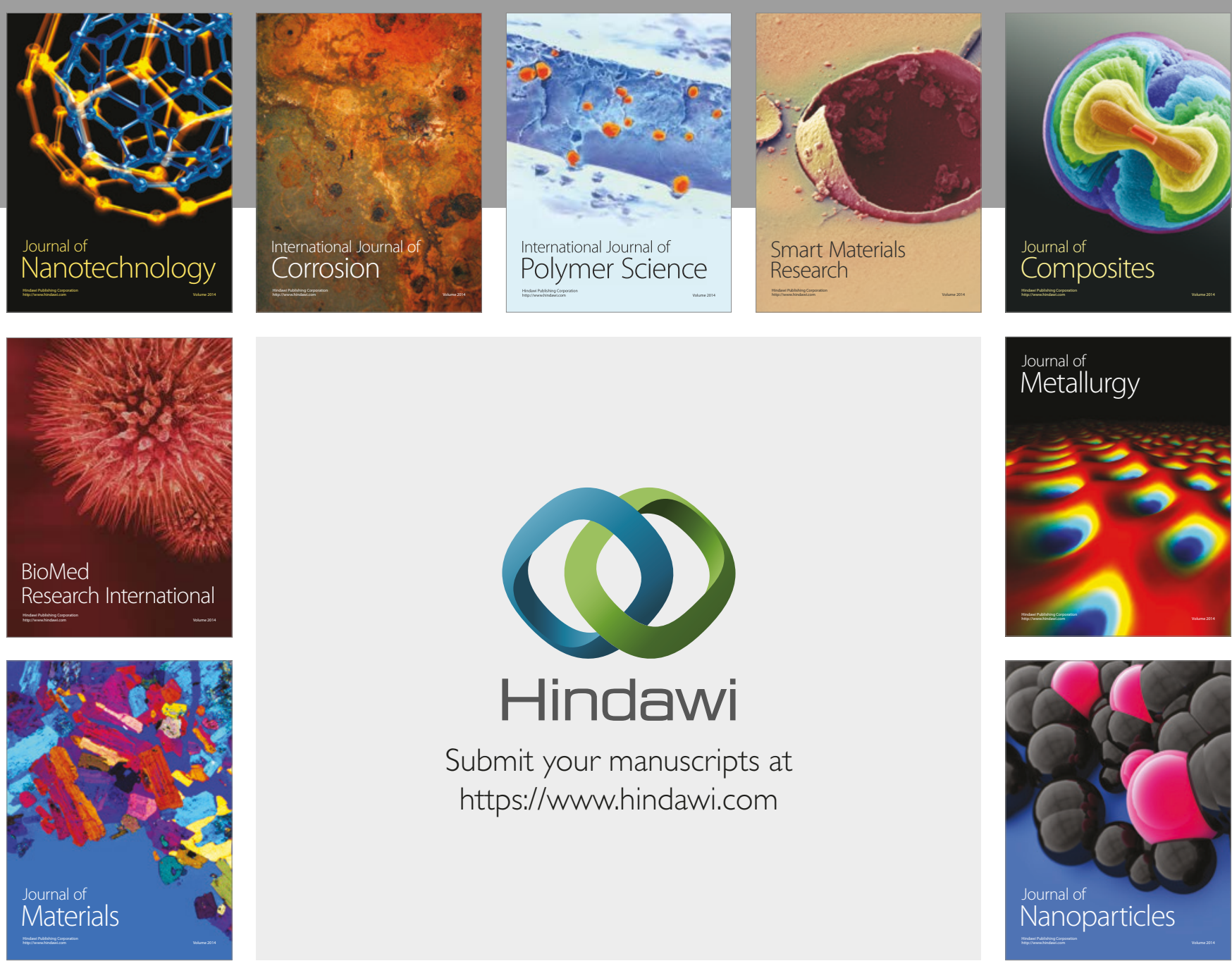

\section{Hindawi}

Submit your manuscripts at

https://www.hindawi.com
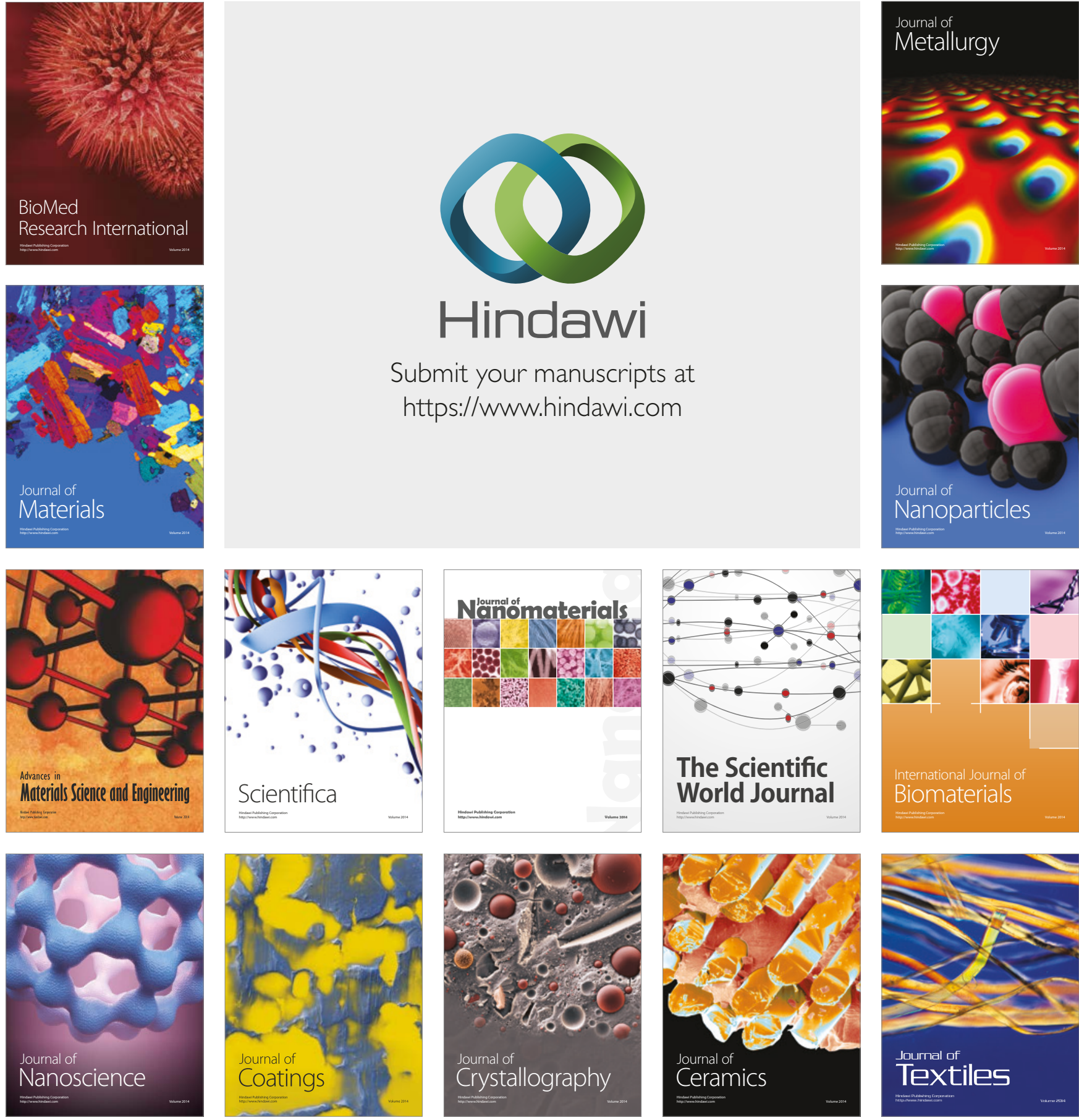

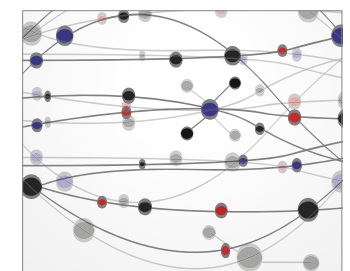

The Scientific World Journal
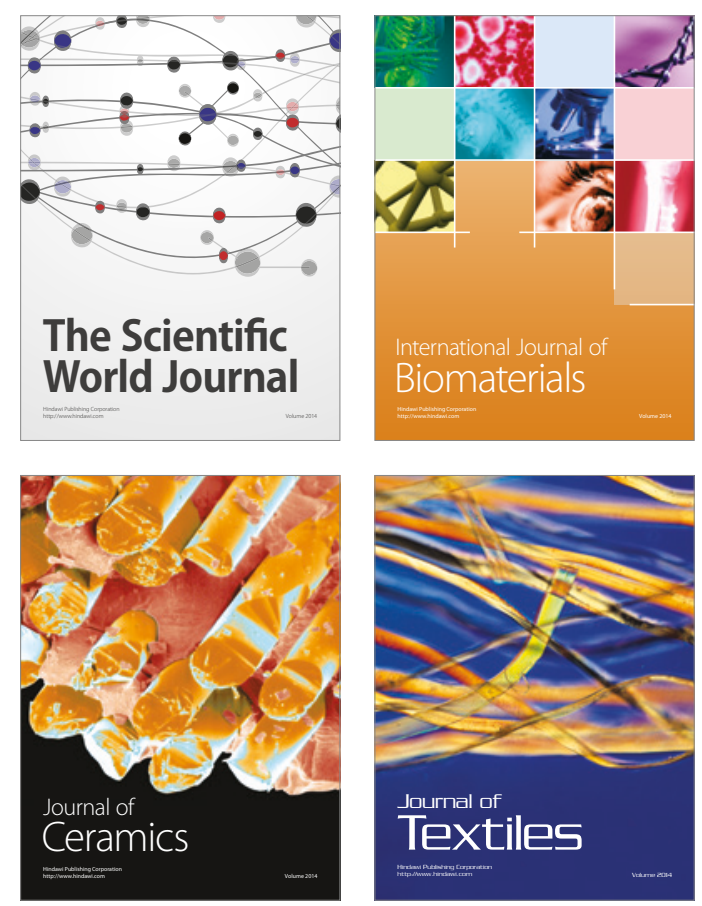